\title{
ESCLAVES ET PLANTATIONS DE SURINAM VUS PAR MALOUET, 1777
}

COMMUNIQUÉ PAR

\section{Gabriel Debien ${ }^{1}$ ) et Johanna Felhoen Kraal}

Dans un des manuscrits de la Bibliothèque de Nantes, dans le Ms. no. 249 , on trouve la copie d'une lettre adressée de Cayenne le 28 décembre 1788 à un correspondant qui habite le Cap-Français à Saint-Domingue. Cette copie de lettre - qui est présentée cidessous - se trouve à la suite d'un Mémoire sur le traitement et l'employ des nègres dans les colonies, fait à Paris en $I 783$ par M. de Malouet, intendant de la Marine à Toulon. La copie est incomplète de quelques expressions et parties de phrases laissées en blanc. Quelques fautes aussi trahissent la main et les yeux d'un étranger qui n'a pu lire certains mots ni développer toutes les abréviations.

1) Noot van de redactie.

GABRIEL DEBIEN (Cairo) houdt zich speciaal bezig met historische studiën betreffende de Franse periode in St. Domingue (Haïti). Van zijn hand zijn de volgende publicaties in de Centrale Boekerij van het Kon. Instituut voor de Tropen aanwezig:

Une plantation de Saint-Domingue: la sucrerie Galbaud du Fort (1690-1802). Notes d'histoire coloniale I, Le Caire, 1941.

Les travaux d'histoire sur Saint Domingue de 1938 à 1946; essai de mise au point. Notes d'histoire coloniale I5, 1947.

Un roman colonial de Victor Hugo: Bug Jargal; ses sources et ses intentions historiques. Notes d'histoire coloniale 22, 1952.

Les femmes des premiers colons des Antilles (1635-1680). Notes d'histoire coloniale 24, 1952.

Les colons de Saint-Domingue et la révolution: essai sur le Club Massiac (Aout I789-Aout I792). La société coloniale aux xvire et xvirie siècles, Paris, 1953.

Esprit colon et esprit d'autonomie à Saint-Domingue au xvirıe siècle. Notes d'histoire coloniale 25, 1954.

Aux débuts d'une grande plantation à Saint-Domingue (1685-1714). Notes d'histoire coloniale 28, 1954.

Colons, marchands et engagés à Nantes au xvire siècle (avec le Dr. L. MERLE). Notes d'histoire coloniale 29, 1954.

Les travaux d'histoire sur Saint-Domingue; chronique bibliographique (1950-1952). Notes d'histoive coloniale 3I, 1953. 
Le nom du destinataire n'est pas donné. Ce n'est certainement pas Stanislas Foäche, très important négociant du Havre et propriétaire de plusieurs plantations à Saint-Domingue, mais peut-être FrancoIs LEgras, ancien procureur général au Conseil du Cap, propriétaire de sucreries à Port-Margot et à Fort-Dauphin qui était l'ami de Malouet et de FoÄche ${ }^{1}$ ). On ne voit pas non plus le nom de celui qui envoie la lettre, mais les sujets abordés nous reportent exactement aux questions qui font l'objet des rapports de MaLOUET sur Surinam et sur la Guyane française et qui ont été publiés dans la Collection des mémoires et correspondances officielles sur l'administration des colonies et notamment sur la Guyane française et hollandaise et dans les Mémoires de Malouet ${ }^{2}$ ). La lettre est de Malouet.

Seule la date laisse perplexe. Il est impossible que la lettre soit du 28 décembre 1788. Malouet avait quitté Cayenne le ler septembre 1778 et avait débarqué à Cherbourg le 11 ou le 12 novembre $\left.{ }^{3}\right)$. Dans le texte il est dit très explicitement que la lettre a été écrite peu après le retour de Surinam. Or c'est vers le 23 septembre 1777 que MaLOuet est revenu à Cayenne. La lettre doit être du mois d'octobre 1777. Rien d'impossible qu'elle soit du 28 décembre 1777.

Si elle porte la date du 28 décembre 1788 c'est par une étourderie de plume du copiste ou par une transposition volontaire et

1) Archives Nationales - Colonies E. 274; et Malouet, Mémoires, 2me édition, I, 35.

Pierre-Victor Malouet (1740-1814), entré dans l'administration de la marine très jeune, inspecteur des embarquements pour les colonies à Rochefort puis à Bordeaux (1764-1765), ordonnateur et commissaire à Saint-Domingue pendant cinq ans. Puis chargé par Sartines d'aller examiner les plans fantastiques du baron de BESSNER qui prétendait améliorer la colonie de la Guyane, il visita Surinam en 1777.

Intendant du port de Toulon, député du tiers de Riom aux Etats Généraux en 1789, fut un des fondateurs du club dit des Impartiaux, et un des conseillers écoutés de Lours xvi. S'enfuit en Angleterre en août 1792. Rentré en 1801, commissaire général de la marine à Anvers en 1803, maitre des requêtes en 1808, conseiller d'Etat en 1810, mort ministre de la marine de la première Restauration.

Il a laissé de nombreux ouvrages, dont un Mémoire sur l'administration de la Marine $\left(1790\right.$, in $\left.8^{\circ}\right)$ et d'importants Mémoires et correspondances officielles sur l'administration des colonies (Paris, 1803, 5 vol. in $8^{\circ}$ ) qui nous ont beaucoup servi ici à établir que le texte de cette lettre était de Malouet.

2) Paris, 2me éd., 1874, 2 vol. in $8^{\circ}$.

3) Mémoires, 2 me éd. I, 460 et 462. 
très précisément dirigée. En cette fin de 1788 certains milieux parisiens sont en pleines discussions sur l'abolition de la traite et de l'esclavage. La Société des Amis des Noirs vient d'être fondée à Paris. Elle est en rapport avec celle de Londres. MaLouET vient de publier ou de laisser publier à Neufchâtel le Mémoire sur l'esclavage des nègres ${ }^{1}$ ) dont il est question dans cette lettre, et son petit livre a fait beaucoup de bruit. LESCALLIER, ordonnateur des colonies, qui arrive de la Guyane, va faire imprimer ses Réflexions sur le sort des Noirs dans nos colonies.

Cette lettre est une arme entre les mains de ceux qui veulent abolir la traite et améliorer le sort des esclaves. Elle démontre le danger permanent qu'est pour la prospérité et même pour la vie coloniale la dureté des planteurs. On a dû vouloir s'en servir dans le débat, et peut-être a-t-on cherché à la publier.

Si l'erreur est due à une simple inadvertance, elle reste pleine de sens pour nous, car elle marque alors probablement la date exacte du travail du copiste, elle demeure un témoignage de la curiosité passionnée que l'on portait à ces questions.

Cette copie nous conserve donc le texte d'une des lettres qui furent détruites par MALOUET au lendemain du 10 août $1792{ }^{2}$ ) ou perdues en 1793 et est un document sur les alentours de la Société des Amis des Noirs à la fin de 1788.

(Bibliothèque de Nantes

Ms. no. 249)

Copie d'une lettre écrite à Saint-Domingue de Cayenne, 28 décembre 1788.

Je n'ai pu, monsieur, avoir l'honneur de vous répondre. Dans le temps où votre lettre m'est parvenue j'arrivai de Surinam avec la fièvre et j'ai été deux mois malade ${ }^{3}$ ). Madame Malouet se chargera de vous marquer ${ }^{4}$ ) combien j'étais sensible à votre souvenir et que je consentais à ce que vous disposassiez du manuscrit qui était entre vos mains, sans en garantir néanmoins l'exactitude. Je ne doute point que vous n'y ayez

1) Mémoire sur l'esclavage des nègres dans lequel on discute les motifs proposés pour leur affranchissement, ceux qui s'y opposent et les moyens praticables pour améliơer leur sort. Neufchâtel, 1788 , in $8^{\circ}, 155 \mathrm{p}$.

$\left.{ }^{2}\right)$ Mémoires, II, 4.

3) Malouet était arrivé à Paramaribo 1 e 10 juillet 1777 . Il était de retour de Surinam vers le 23 septembre (Mémoires, I, 168 et 162 note 2). Il avait eu la fièvre tout le mois de septembre (id. I, 161).

4) Madame Malouet, qui était originaire du Cap-Français à SaintDomingue, aurait-elle été alors au Cap? Elle avait accompagné son mari à Surinam. Elle rentrera avec lui en France. 
suppléé et que si vous l'in $\left.\ldots . . .{ }^{1}\right)$ L'époque où cet écrit ${ }^{2}$ ) a été présenté au roi et au conseil en a seul fait le mérite. Il y avait alors une déviation de principes en plus d'un genre qui aurait pu nous mener loin. Les Economistes en eurent connaissances. Il fut lu à leur assemblée ${ }^{3}$ ). On menaça d'y répondre victorieusement. Cependant la réponse ne parut point. On se réduisit à des notes marginales de la main de M. DE Mirabeau, ${ }^{4}$ ) que je garde précieusement. Il me convenait fort alors de rester inconnu, et lorsque les circonstances changèrent, le moment de crise étant passé, je ne jugeai pas alors de revenir sur cet objet qui avait eu dans le temps tout son effet. En écrivant sur l'esclavage et sur la nécessité de le maintenir dans nos colonies, je n'ai pas dit, à beaucoup près, tout ce qu'un sujet pouvait me fournir, ou plutôt j'ai renvoyé à une autre circonstance la démonstration des moyens nécessaires pour concilier en cette partie l'humanité et la politique. Je suis très persuadé, monsieur, que nous avons des devoirs à remplir envers nos esclaves, dont le plus grand nombre des maitres s'affranchit, et qu'il est de l'intérêt du gouvernement et de celui des particuliers d'y veiller avec plus d'exactitude qu'on ne l'a jamais fait. Si on n'adoucit la condition de l'esclave, si on ne lui inculque la portion de morale et de religion dont il est susceptible, si le despotisme domestique et ses excès ne sont repoussés, si on ne met un frein à la licence ...... qui en résulte, nos colonies éprouveront les mêmes révolutions que Surinam. ${ }^{5}$ ) Dans tous les pays, dans tous les temps, les mêmes causes ont produit les mêmes effets, et il ne faut pas attendre que chaque propriétaire ou régisseur soit retenu par la réflexion et la conscience de ses désirs. Ne nous dissimulons pas que cette distance d'un homme à un homme, l'habitude et le pouvoir de faire à chaque instant sa volonté en dérègle les mouvements, dégrade les caractères, qu'il faut une excellente éducation et des principes hien ótablis pour rósistor d cecle impulsion et

1) Faudrait-il compléter en: "imprimez"?

$\left.{ }^{2}\right)$ Il s'agit apparemment du Mémoire sur l'esclavage des nègres qui sera publié en 1788, ou du Mémoire sur le traitement et l'employ des nègres dans les colonies, qui parait en être une première ébauche, et dont une copie se trouve à côté de la copie de cette lettre parmi les manuscrits de la Bibliothèque de Nantes.

$\left.{ }^{3}\right)$ De quelle société serait-il ici question? Nous savons qu'un rapport de Malouet sur la Guyane, de 1776, fut examiné par Condillac (Collection des Mémoires, $\mathrm{I}, 158)$.

4) Nous savons par Malouet (Mémoires I, 40) que son Mémoire sur l'esclavage publié en 1788 fut accompagné de notes de Mirabeau. Il s'agit donc ici de ce Mémoire.

$\left.{ }^{5}\right)$ Les marrons, esclaves fugitifs, qui vivaient dans l'intérieur du pays depuis près d'un siècle étaient renforcés continuellement par d'autres fugitifs. Formant des bandes ils faisaient la guerilla contre les plantations. L'expédition dont faisait partie Stedman fut organisée par le gouverneur. Cf. J. G. Stedman, Narrative of a Five Years' Expedition against the Revolted Negroes of Surinam in Guiana, 1772-1777, London, 1796, 2 vol. in $8^{\circ}$, traduit en français sous le titre de Voyage à Surinam et dans l'intérieur de la Guiane par le capitaine J. G. STEDMan, traduit de l'Anglais par P. F. Henry; suivi du Tableau de la colonie trançaise de Cayenne, Paris, an viI, 3 vol. in $8^{\circ}$. 
que le plus grand nombre des hommes n'en est pas capable. Comment donc pourrait-on se passer d'un contrepoids? Je n'en vois point d'autre que la religion pour les esclaves ....... et l'oeil de l'administration pour les maîtres qui leur en imposent et les arrêtent dans leurs écarts. J'ai prêché ces vérités à Surinam et elles ont été senties. J'ai vu les Hollandais regretter pour leurs nègres la proscription du culte romain ${ }^{1}$ ) et $y$ tenter la voie des missions. J'ai vu convenir unanimement de la nécessité d'une police sévère pour les maîtres qui abusent, et on m'a donné la satisfaction pendant mon séjour de bannir de la colonie un blanc qui avait donné un coup de couteau à sa négresse. Mon opinion sur cette matière se trouvait confirmée dans leur pays même par des exemples frappants. Dans le grand nombre d'habitants ${ }^{2}$ ) que j'ai visités j'en ai trouvé plusieurs dont la police est [soit] supérieure à la nôtre, où les nègres sont plus heureux, plus soignés que parmi nous et jamais dans ces ateliers on n'en a eu de marrons ${ }^{3}$ ). J'ai passé trois jours chez une madame Godefroy ${ }^{4}$ ) qui a cinq cents nègres. La grande punition de ses

1) Le culte catholique était officiellement interdit pendant le régime de la Societeit van Suriname et de facto jusqu'en 1817. Des missionnaires protestants (de l'église morave) travaillaient parmi les Indiens depuis 1735 et parmi les marrons depuis 1765 . Leur mission auprès des esclaves avait commencé en 1776 .

$\left.{ }^{2}\right)$ Il faut lire: habitations.

3) C'était le meilleur indice de la bonne discipline et du bon esprit d'un atelier.

4) Tout ce qui concerne ici l'histoire de Madame Godefroy et celle des maitres de BARoN se trouve en abrégé presque littéralement dans le Compte rendu (Collection des Mémoires, tome III, p. 43/44) tandis que la première phrase de cet alinéa indique également ce qui est dit dans la lettre sur la nécessité d'un culte pour les esclaves: "Quant aux observations insérées dans mon mémoire sur la police domestique, sur la nécessité d'un culte pour les esclaves, on doit bien présumer que les conférences antécédentes y avoient donné lieu. Les membres de la régence, ainsi que les habitants, m'avoient appris des traits horribles de cruauté presque toujours impunis; j'avois déjà visité plusieurs habitations, et j'avois vu sur celles qui sont régies avec humanité, qu'on n'y avoit jamais éprouvé aucun des désordres du marronnage. J'avois vu chez madame Godefroy cinq cens esclaves ne connoître d'autre bonheur que celui de la servir, et son atelier gémissant sur le sort d'un domestique qu'elle avoit par punition chassé de sa présence. J'avois vu la maîtresse du célèbre Baron, capitaine des marrons ennemis, éprouvant de la part de son esclave révolté les marques les plus touchantes de respect et d'attachement. Ce nègre n'en vouloit qu'à son maître, qui l'avoit traité avec cruauté: il est venu dix fois sur sa terre avec le projet de tout incendier; mais la maîtresse et ses enfans étoient pour lui une sauvegarde qu'il respectoit. Il se jetoit à leurs pieds, embrassoit ses petits maîtres, et s'en retournoit sans faire aucun mal, quand il voyoit que monsieur étoit absent. Enfin, depuis que j'habite les colonies, j'ai assez vu, assez réfléchi sur le caractère et la position respective des maîtres et des esclaves, pour être convaincu de la nécessité de surveiller les uns et les autres et de les contenir. Si nous n'avons jamais eu dans nos colonies de guerre à soutenir 
esclaves est la défense de voir leur maîtresse et de se trouver sur son chemin quand elle passe. Il n'y en a pas un qui ne préfère cent coups de fouet à cette excommunication. L'un de ces exilés profita de mon passage pour obtenir sa grâce. Lui et toute sa famille fondaient en larmes. Cette madame Godefroy s'est trouvée plusieurs fois dans le cas d'alarmes comme les autres habitants, mais ses nègres en imposent tellement aux marrons ennemis qu'on n'a jamais osé faire la moindre incursion chez elle. Il y a trente habitants qui sont dans la même sécurité, respectée même des troupes ennemies. Le chef de la dernière bande nommé BARON, ${ }^{1}$ ) traité avec cruauté par son maître, avait conçu un tel atta-

contre les esclaves, la douceur de nos moeurs d'une part, et la religion de l'autre, en sont les seules causes. Il est vrai qu'elles tendent incessamment à s'affoiblir, que nous avons aussi quelques exemples de férocité non reprimée, et que l'autorité publique néglige beaucoup trop la police domestique et le culte religieux; il en résulteroit à la longue les mêmes malheurs qu'éprouvent les Hollandais et les Anglais: ceci soit dit en passant et pour mémoire; car je me reprocherois éternellement d'avoir écrit sur l'emploi et l'esclavage des nègres, si un seul homme pouvoit me croire fauteur et complice de ses abus".

Voici en quels termes STEDMAN parle de cette dame: "Je dois rendre témoignage des vertus sublimes de mesdames Elisabeth Danforth et Godefroy [dans le texte anglais on lit: Mrs. Elizabeth Danforth now Mrs. Godefrey] et de quelques autres dont le caractère est sans taches" (Voyage à Surinam, II, 216). Puis après un récit enthousiaste de la façon dont Mme Godefroy lui a prêté 2000 florins pour racheter la liberté de l'esclave dont il avait eu un enfant, et de cet enfant. Stedman ajoute (p. 251): "L'humanité de cette dame ne se borna pas à ne rendre service qu'à nous, car ayant appris la situation déplorable des malades au mont Magdenberg, elle leur envoya en présent une barge chargée de fruits, de végétaux et de rafraîchissements de toutes sortes".

1) "Baron avait été esclave d'un Suédois nommé M. Dahlbergh, qui l'avait distingué en raison de son adresse et de son intelligence. Il lui avait fait aussi apprendre à lire et à écrire, et le métier de maçon. L'esclave avait accompagné son maître en Hollande, et celui-ci lui avait promis sa liberté à son retour dans la colonie. Mais il manqua à sa parole et vendit Baron à un juif. Le nègre refusa obstinément le travail et fut en conséquence fustigé publiquement au pied d'une potence. Il en fut si outré, que de ce moment il ne songea plus qu'à se venger de tous les Européens sans exception. Il s'enfuit dans les bois où il se mit à la tête des rebelles; son nom répandit l'épouvante, et il jura de ne jamais poser les armes qu'il n'eût trempé ses mains dans le sang de M. Dahlbergh, son tyran" (Collection des Mémoires, I, p. 110 et 114).

Voir également Oudschans Dentz Het einde van de legende DahlbergBaron (West Indische Gids x, 1928, p. 165-167), où est publiée l'attestation de la Cour de Justice, d'août 1777, constatant que l'esclave BARON n'avait jamais été emmené hors de Surinam, qu'il avait été vendu "pour mauvais comportement" par M. DAHLBERG à un autre maître, non pas juif, mais chrétien, qui l'avait revendu à un maçon de chez qui Baron s'était échappé. Tous les on-dit au sujet des mauvais traitements de la part de Dahlberg seraient "destitués de tout fondement." 
chement pour sa maîtresse et ses enfants, qu'étant venu onze fois avec ses gens, la torche à la main pour incendier l'habitation, lors qu'on lui disait que Monsieur n'y était pas, qu'il n'y avait que Madame et ses enfants, il allait se jeter à leurs pieds et les caressait et s'en retournait sans leur faire aucun mal. Voilà donc les Hollandais convaincus que l'inconduite, la dureté de plusieurs régisseurs ou propriétaires et le défaut de culte parmi leurs esclaves ont occasionné les désertions fréquentes dont ils ont à se plaindre et les dépenses ruineuses auxquelles ils sont aujourd'hui contraints pour la garde de leurs colonies. Parmi nous les individus nationnals [sic] ...... et l'association de nos esclaves au culte que nous professons, nous ont garanti des mêmes accidents, mais d'une part les individus dégénèreront promptement dans les colonies et de l'autre l'empire et les pratiques de la religion s'y affaiblissent encore plus sensiblement. C'est donc à l'administration à tendre les ressorts qui se relâchent et c'est aux colons sages et éclairés qu'il appartient d'en sentir et faire connaitre la nécessité! Vous me demandez, monsieur, de vous faire part des observations utiles que j'aurai pu recueillir. Si je pouvais vous envoyer mon portefeuille vous en trouveriez quelques unes d'intéressantes pour les colonies en général, mais je suis si continuellement occupé dans l'intervalle de mes voyages et de mes maladies qu'il me reste fort peu d'instants à donner aux correspondances qui me plairaient le plus.

J'ai parcouru toute la Guyane française et hollandaise. J'ai vu sur le même sol et dans un même climat les résultats nécessaires de l'ordre, de l'industrie, de l'ignorance et de la folie.

J'ai vu sur notre territoire une vaste et superbe possession destinée par la nature à un grand commerce et à de riches cultures mais condamnée à la stérilité par l'impéritie de ses habitants et par l'absurdité plus criminelle des gens à projets. ${ }^{1}$ ) Les Hollandais m'ont, au contraire, présenté le plus magnifique spectacle que puisse produire le courage et l'industrie. Une armée de 100.000 hommes en bataille et la colonie de Surinam sont les deux choses que je me félicite le plus d'avoir vues. Je me suis promené dans des jardins plus beaux que ceux des Tuileries, qui étaient il y a dix ans couverts de dix pieds d'eau ${ }^{2}$. Notre terre de SaintDomingue est en général supérieure à la leur et par sa qualité et parce qu'elle n'exige pas la vingtième partie des travaux nécessaire à leur exploitation, mais leurs procédés de culture sont bien mieux entendus, mieux ordonnés que les nôtres. Ce qui m'a paru surtout admirable c'est l'uniformité d'ordre et de méthode dans la distribution et l'exécution des travaux. Toutes les habitations se ressemblent. Les ateliers ne sont ni plus ni moins nombreux, les bâtiments sont plus ou moins riches, mais tous calqués sur le même plan. Ce n'est point le hasard qui a placé là les

1) Allusion à l'aventưre du Kourou et aux chimériques conceptions du baron de BESSNER relativement aux Indiens.

$\left.{ }^{2}\right)$ “...... Quatre cents habitations contiguës, travaillées sur le même plan, présentant le même ensemble d'ordre, de vues et de moyens; lorsqu'enfin je me suis vu sur une de ces habitations nouvellement sorties de dessous l'eau, parcourant des jardins aussi vastes, aussi bien dessinés que les Tuileries; des terrasses aussi bien nivelées que celles de Bellevue ......" Collection des Mémoires, III, 91. 


\section{ESCLAVES ET PLANTATIONS DE SURINAM VUS PAR MALOUET}

habitants ni leur caprice qui les a dirigés. Ils sont tous subordonnés à l'exécution du même plan qui a été dans l'institution de la colonie. Des ingénieurs habiles ont tracé le premier modèle des travaux de dessèchement et chaque particulier a été obligé de le suivre. La baisse des cafés et le faste dans les bâtiments et les jardins ont ruiné la majeural [sic] partie des propriétaires, mais la colonie est florissante. Les biens passent journellement des débiteurs aux créanciers et lorsque la révolution sera consommée, lorsque les gens ruinés auront disparu, on ne s'apercevra d'aucun changement dans les produits. La police y est active et sévère. Les principes y sont républicains et les formes monarchiques, ce qui forme la combinaison de gouvernement la plus efficace.

Je ne vous parle point de Cayenne parce qu'il faudrait vous en trop parler. Voici le précis très succint de ce que j'y ai fait. Vous concluerez de là qu'il y a eu de ma part un développement de vues et de moyens qui forme un complément de ma mission. Elle m'a plu infiniment. J'y ai trouvé pâture à mon activité et j'y ai plus écrit peut-être sur l'administration de Cayenne que tous les administrateurs qui m'ont précédé. ${ }^{1}$ ) Cependant je me suis borné à constater les faits et à démontrer quelques vérités. J'y ai joint la leçon et l'exemple d'un dessèchement de terres basses exécuté par un ingénieur hollandais que j'ai amené de Surinam ${ }^{2}$ ) et tout cela pour produire à la longue un mouvement d'idées et de travaux utiles.

Je ne m'ennuie point comme vous voyez de causer avec vous, mais il faut clore mes paquets. Adieu, monsieur, j'espère avoir le plaisir de vous voir vers le mois d'avril à mon passage au Cap et vous y renouveler les assurances des sentiments que je vous ai voués et avec lesquels ......

1) Le Postillon de Cayenne, qui ramenait Malouet et les siens en France fut attaqué et pris le 22 octobre 1778 par un corsaire anglais qui le conduisit à Weymouth. Malouet obtint un passeport pour rentrer en France mais "les caisses contenant ses collections, ses papiers, ses documents, tout fut pris, rien ne fut rendu"' (Mémoires, $I, 457)$. Cependant aux Archives de la Marine, à Toulon (5 R.I.) il y a un dossier sur la mission de Malouet à la Guyane, qui paraît avoir été ramené de Cayenne.

$\left.{ }^{2}\right)$ Ce ne peut être que le chevalier Guisan que Malouet en effet ramena de Surinam et fit nommer directeur des travaux de desséchement. Mais Guisan n'était pas Hollandais, mais Suisse, cf. Charles Eynard, Le Chevalier Guisan, sa vie, et ses travaux à la Guyane, Paris, 1844 , in $8^{\circ}$. 